\title{
AQUISIÇÃO DE HABILIDADES E COMPETÊNCIAS PARA CUIDAR EM SAÚDE MENTAL: AUTOAVALIAÇÃO DE ESTUDANTES DE ENFERMAGEM
}

\section{ACQUISITION OF CARE-GIVING SKILLS AND COMPETENCIES IN MENTAL HEALTH: NURSING STUDENT SELF- ASSESSMENT}

\section{ADQUISICIÓN DE HABILIDADES Y COMPETENCIAS PARA CUIDAR LA SALUD MENTAL: AUTOEVALUACIÓN DEL ESTUDIANTES DE ENFERMERÍA}

Aline Mesquita Lemos ${ }^{1}$, Helder de Pádua Lima², Maria Dalva Santos Alves ${ }^{3}$, Ângela Maria Alves e Souza ${ }^{4}$, Maria Isis Freire de Aguiar ${ }^{5}$, Michell Ângelo Marques Araújo ${ }^{6}$.

\section{RESUMO}

Objetivo: identificar como estudantes de enfermagem avaliam a aquisição de habilidades e competências para o cuidado em saúde mental. Métodos: estudo descritivo, transversal, realizado com 73 estudantes de enfermagem. Os dados foram coletados, por meio de questionário e apresentados em tabelas. Analisou-se a associação entre períodos de curso, observação da prática do cuidado de enfermagem em saúde mental, e aquisição de habilidades e competências para tal prática, por meio dos testes qui-quadrado de Pearson e razão de verossimilhança. Resultados: houve associação entre períodos de graduação em curso e observação das práticas de lidar com paciente em crise $(\mathrm{p}=0,064)$ e aplicar a Assistência de Enfermagem em Saúde Mental no contexto comunitário ( $p=0,097)$; a aquisição das habilidades de reconhecer preceitos da Política Nacional de Saúde Mental $(p=0,021)$ e aplicar a Sistematização da Assistência de Enfermagem junto à pessoa com transtorno mental $(p=0,051)$; e a aquisição das competências caracterizar transtornos mentais $(p=0,013)$ e atuar no nível de atenção primária à saúde mental $(p=0,001)$. Conclusão: diante das lacunas identificadas na aquisição de habilidades e competências para o cuidado de enfermagem, em saúde mental, por parte de estudantes, ressalta-se a necessidade de reforçar a formação em enfermagem em saúde mental por competências e habilidades.

Descritores: Enfermagem; Saúde mental; Estudantes de enfermagem; Competência Profissional; Enfermagem Psiquiátrica.

\begin{abstract}
Objective to investigate how nursing students evaluate the acquisition of care-giving skills and competencies in mental health. Methods: cross-sectional descriptive study based on a sample composed of 73 nursing students. Data were collected through questionnaire application and presented in tables. Association among course semester, observation of nursing-care practice in mental health and acquisition of skills and competencies for such practice were analyzed through Pearson's chi-square and likelihood ratio tests. Results: there was association between undergraduate course semesters and observation of practices to deal with patients in crisis $(p=0.064)$ and to apply Mental Health Nursing Care in communities ( $p=0.097)$; between the acquisition of skills to identify precepts of the National Mental Health Policy $(p=0.021)$ and to apply the Nursing Care Systematization to patients with mental disorder $(p=0.051)$; and between the acquisition of skills to characterize mental disorders $(p=0.013)$ and to act at primary mental healthcare level $(p=0.001)$. Conclusion: given the gaps identified in the students' acquisition of nursing-care skills and competencies in mental health, it is important to emphasize the need to improve nursing students' training by developing their skills and abilities in mental health.
\end{abstract}

Descriptors: Nursing; Mental Health; Students, Nursing; Professional Competence; Psychiatric Nursing.

\section{RESUMEN}

Objetivo: Identificar como los estudiantes de enfermería evalúan la adquisición de habilidades y competencias para la atención de la salud mental. Métodos: Estudio descriptivo de corte transversal con 73 estudiantes de enfermería. Los datos fueron recolectados a través de un cuestionario y presentados en tablas. Se analizaron las asociaciones entre los períodos del curso, la observación de la práctica de la atención de enfermería en salud mental y la adquisición de habilidades y competencias para esta práctica mediante la prueba de chi-cuadrado de Pearson y la razón de probabilidad. Resultados: Hubo una asociación entre los períodos de pregrado y la observación de las prácticas para tratar pacientes en crisi $(p=0.064)$ y la aplicación de la Asistencia de Enfermería en Salud Mental en el contexto comunitario $(p=0.097)$; la adquisición de las habilidades para reconocer los preceptos de la Política Nacional de Salud Mental $(\mathrm{p}=0.021)$ y aplicar la Sistematización de la Asistencia de Enfermería junto a la persona con trastorno mental ( $p=0.051)$; y la adquisición de habilidades para caracterizar los trastornos mentales $(p=0.013)$ y actuar en el nivel de atención primaria de salud mental $(\mathrm{p}=0.001)$. Conclusión: Dadas las lagunas identificadas en la adquisición de habilidades y competencias para la atención de enfermería en salud mental por parte de los estudiantes, se enfatiza la necesidad de reforzar la formación en enfermería de la salud mental por competencias y habilidades.

Descriptores: Enfermería; Salud Mental; Estudiantes de Enfermería; Competencia Professional; Enfermería Psiquiátrica.

${ }^{1}$ Enfermeira. Mestre em Enfermagem pela Universidade Federal do Ceará. Docente na UniAteneu. ${ }^{2}$ Professor Adjunto do Curso de Enfermagem da UFMS. Doutor em Enfermagem pela UFC. ${ }^{3}$ Enfermeira. Doutora em Enfermagem pela Universidade Federal do Ceará. Docente na Universidade Federal do Ceará. ${ }^{4}$ Enfermeira. Doutora em Enfermagem pela Universidade Federal do Ceará. Docente na Universidade Federal do Ceará. ${ }^{5}$ Enfermeira. Doutora em Enfermagem. Docente na Universidade Federal do Ceará. ${ }^{6}$ Enfermeiro. Doutor em Enfermagem pela Universidade Federal do Ceará. Docente na Universidade Federal do Ceará.

\section{Como citar este artigo:}

Lemos AM, Lima HP, Alves MDS, et al. Aquisição de habilidades e competências para cuidar em saúde mental: autoavaliação de estudantes de enfermagem. Revista de Enfermagem do Centro-Oeste Mineiro. 2019;9:e3476. [Access in: DOI: http://dx.doi.org/10.19175/recom.v9i0.3476 


\section{INTRODUÇÃO}

A passagem da institucionalização para a inserção social daqueles que sofrem com transtornos mentais tem exigido da assistência do enfermeiro uma integração de saberes e práticas que permita reconhecer a pessoa em uma realidade social e em um contexto familiar. Esse reconhecimento requer, na formação desse profissional, a adoção de um modelo de atenção em saúde mental, pautado na superação do modelo biomédico e hospitalocêntrico/manicomial, que valorize aspectos biopsicossociais da atenção à saúde e demarque um compromisso com os princípios do Sistema Único de Saúde (SUS). No entanto, vários autores afirmam que a formação de enfermeiros para atuar em saúde mental tem sido deficitária, em decorrência das indefinições quanto às habilidades e às competências necessárias para sua atuação nesse campo ${ }^{(1-2)}$.

De acordo com as Diretrizes Curriculares Nacionais (DCN), o processo formativo do estudante de enfermagem deve estar pautado nas competências específicas da profissão, considerando o discente protagonista, na perspectiva de tornar-se um profissional generalista, humanista, crítico e reflexivo, incluindo, nesse contexto, a atenção à saúde mental ${ }^{(3-4)}$.

As DCN orientam-se pela pedagogia das competências como forma de superar o enfoque descontextualizado e disciplinar do ensino. Referese a competências como domínios práticos das situações cotidianas que, necessariamente, passam pela compreensão da ação empreendida e do uso a que essa ação se destina. Elas só podem ser alcançadas, se forem desenvolvidas em conjunto com as habilidades dos estudantes, o que só se pode realizar a partir da compreensão do conteúdo que explica aquele domínio(5).

No Brasil, ainda não há consenso nem regulamentação acerca das competências específicas para a prática do enfermeiro em saúde mental. Há, contudo, certa concordância nacional de docentes de enfermagem, desse campo, que o ensino deva ser orientado pelos princípios da Reforma Psiquiátrica. Nessa perspectiva, esses docentes acreditam que ao organizarem e desenvolverem o planejamento do ensino estarão formando enfermeiros competentes e hábeis para a prática assistencial em saúde mental ${ }^{(5-6)}$.

Somada a essa indefinição, outros fatores influenciam de modo a dificultar o estudante de apreender e colocar em prática o conhecimento adquirido e levá-lo para sua vida profissional, dentre eles: a predisposição e a escolha discente por outras áreas de interesse; particularidades nas metodologias utilizadas por cada docente; a imaturidade de parte de estudantes que ingressam, cada vez mais cedo, nas universidades; a falta de formação específica e a falta de conhecimento na íntegra do currículo, por parte de alguns docentes; os saberes e as práticas de enfermagem em saúde mental não serem ministrados de forma transversalizada em muitos currículos; o pouco tempo de contato do aluno com a temática; e a rotatividade de professores substitutos ${ }^{(7)}$.

Com base no exposto e na experiência docente em enfermagem em saúde mental, em uma universidade pública, cujo Projeto Político Pedagógico delimita habilidades e competências específicas em saúde mental, optou-se pelo desenvolvimento, deste estudo, que partiu do seguinte questionamento: como estudantes de enfermagem avaliam a aquisição de habilidades e competências para o cuidado em saúde mental?

De modo a responder a esse questionamento, esse artigo objetivou identificar como estudantes de enfermagem avaliam a aquisição de habilidades e competências para o cuidado em saúde mental.

\section{MÉTODOS}

Trata-se de um estudo descritivo e transversal, realizado com estudantes de enfermagem de uma universidade pública localizada no nordeste do Brasil. A população foi representada por 82 estudantes regularmente matriculados no semestre letivo 2016.1. Os critérios de inclusão foram: estar regularmente matriculado no sexto, sétimo ou oitavo períodos do curso de graduação em enfermagem. Foram excluídos aqueles que efetivaram trancamento institucional, no período da coleta e que se recusaram a participar da pesquisa. A amostra foi composta por 73 alunos, sendo: 19 do sexto período, 33 do sétimo e 21 do oitavo.

Optou-se por tais períodos de curso, pois eram neles em que conteúdos teóricos e práticos de Enfermagem em Saúde Mental eram abordados com maior ênfase, a saber: sexto período (disciplina 'Enfermagem no Processo de Cuidar em Saúde Mental'), sétimo período (disciplinas 'Enfermagem no Processo de Cuidar do Idoso' e 'Enfermagem no Processo de Cuidar da Criança na Atenção Básica') e oitavo período (disciplinas 'Enfermagem no Processo de Cuidar 
da Saúde Sexual e Reprodutiva' e 'Enfermagem no Processo de Cuidar da Criança no contexto hospitalar'). Ressalta-se que, além das aulas teóricas nas disciplinas mencionadas, os estudantes tinham a oportunidade de observar docentes de enfermagem executando o cuidado de enfermagem em saúde mental e realizar essa prática em laboratórios e serviços públicos de saúde (Unidades Básicas de Saúde, Centros de Atenção Psicossocial, hospital-dia, hospitais clínico e psiquiátrico).

A coleta de dados ocorreu, em junho de 2016, por meio de um questionário que abordava aspectos sociodemográficos dos estudantes (sexo, idade, naturalidade, estado civil, número de filhos, profissão/ocupação, renda individual e familiar) e uma autoavaliação com relação à aquisição de habilidades e competências para a realização do cuidado de enfermagem em saúde mental, e observação da execução da prática por um docente do curso. Foram consideradas as seguintes habilidades e competências específicas para o cuidado de enfermagem em saúde mental, propostas no Projeto Pedagógico do Curso da referida instituição: reconhecer preceitos da Política Nacional de Saúde Mental; aplicar o exame do estado mental; desenvolver o relacionamento terapêutico; coordenar grupos; caracterizar transtornos mentais; aplicar a Sistematização da Assistência de Enfermagem (SAE) junto à pessoa com transtorno mental; lidar com o paciente em crise de transtorno mental; atuar nos níveis de atenção primária, secundária e terciária à saúde mental; e aplicar a Assistência de Enfermagem em Saúde Mental nos contextos individual, familiar e comunitário.

Os dados foram organizados e processados utilizando-se o programa Software Statistical
Package for the Social Sciences (SPSS) versão 20.0, licença no 10101131007. Os resultados foram apresentados em tabelas com frequências absolutas e relativas. Analisou-se a associação entre períodos de curso, a observação da prática do cuidado de enfermagem em saúde mental e a aquisição de habilidades e competências para tal prática por meio dos testes qui-quadrado de Pearson e razão de verossimilhança. Para todas as análises, $o$ valor de $p<0,05$ foi considerado estatisticamente significante.

A pesquisa foi aprovada pelo Comitê de Ética em Pesquisa, da Universidade Federal do Ceará, no protocolo $\mathrm{n}^{\circ} 480.404$, CAAE $\mathrm{n}$ 20166013.5.0000.5054, e respeitou os aspectos éticos e legais previstos na Resolução №466/2012 do Conselho Nacional de Saúde ${ }^{(8)}$.

\section{RESULTADOS E DISCUSSÃO}

Com relação ao perfil dos participantes, predominaram estudantes do sexo feminino (91,8\%), na faixa etária entre 19 e 24 anos $(71,2 \%)$, naturais de Fortaleza - Ceará $(71,2 \%)$, solteiros $(91,8 \%)$, sem filhos $(91,8 \%)$, que se dedicavam apenas aos estudos (53,3\%), com renda individual de até 1 (um) salário mínimo $(68,5 \%)$ e renda familiar entre dois e quatro salários mínimos (40,0\%).

De acordo com a Tabela 1, em síntese, houve associação entre períodos de graduação em curso e aquisição da competência caracterizar transtornos mentais $(p=0,013)$, aquisição das habilidades reconhecer preceitos da Política Nacional de Saúde Mental $(p=0,021)$ e aplicar a SAE junto à pessoa com transtorno mental $(p=0,051)$ e observação da prática de lidar com paciente em crise $(p=0,064)$.

Tabela 1 - Distribuição dos estudantes de acordo com período de graduação em curso e autoavaliação com relação à observação da execução do cuidado de enfermagem em saúde mental por um docente do curso, e aquisição de habilidades e competências para realização do cuidado de enfermagem em saúde mental. Fortaleza, CE, Brasil, 2016. ( $\mathrm{N}=73$ ).

\begin{tabular}{|c|c|c|c|c|c|c|c|}
\hline \multirow{3}{*}{ Variáveis } & \multicolumn{6}{|c|}{ Período de graduação em curso } & \multirow{3}{*}{$\begin{array}{l}\text { Valor } \\
\text { de } \mathrm{p}^{*}\end{array}$} \\
\hline & \multicolumn{2}{|c|}{$\begin{array}{l}\text { Sexto } \\
(N=19)\end{array}$} & \multicolumn{2}{|c|}{$\begin{array}{l}\text { Sétimo } \\
(\mathrm{N}=33)\end{array}$} & \multicolumn{2}{|c|}{$\begin{array}{l}\text { Oitavo } \\
(\mathrm{N}=21) \\
\end{array}$} & \\
\hline & $\mathbf{N}$ & $\%$ & $\mathbf{N}$ & $\%$ & $\mathbf{N}$ & $\%$ & \\
\hline \multicolumn{8}{|c|}{$\begin{array}{l}\text { Reconhecer preceitos da Política Nacional de Saúde Mental } \\
\text { Observação da prática }\end{array}$} \\
\hline Sim & 17 & 89,5 & 28 & 84,8 & 18 & 85,7 & 0,893 \\
\hline Não & 2 & 10,5 & 5 & 15,2 & 3 & 14,3 & \\
\hline \multicolumn{8}{|l|}{ Aquisição da habilidade } \\
\hline Sim & 16 & 84,2 & 15 & 45,5 & 11 & 52,4 & 0,021 \\
\hline Não & 3 & 15,8 & 18 & 54,5 & 10 & 47,6 & \\
\hline
\end{tabular}




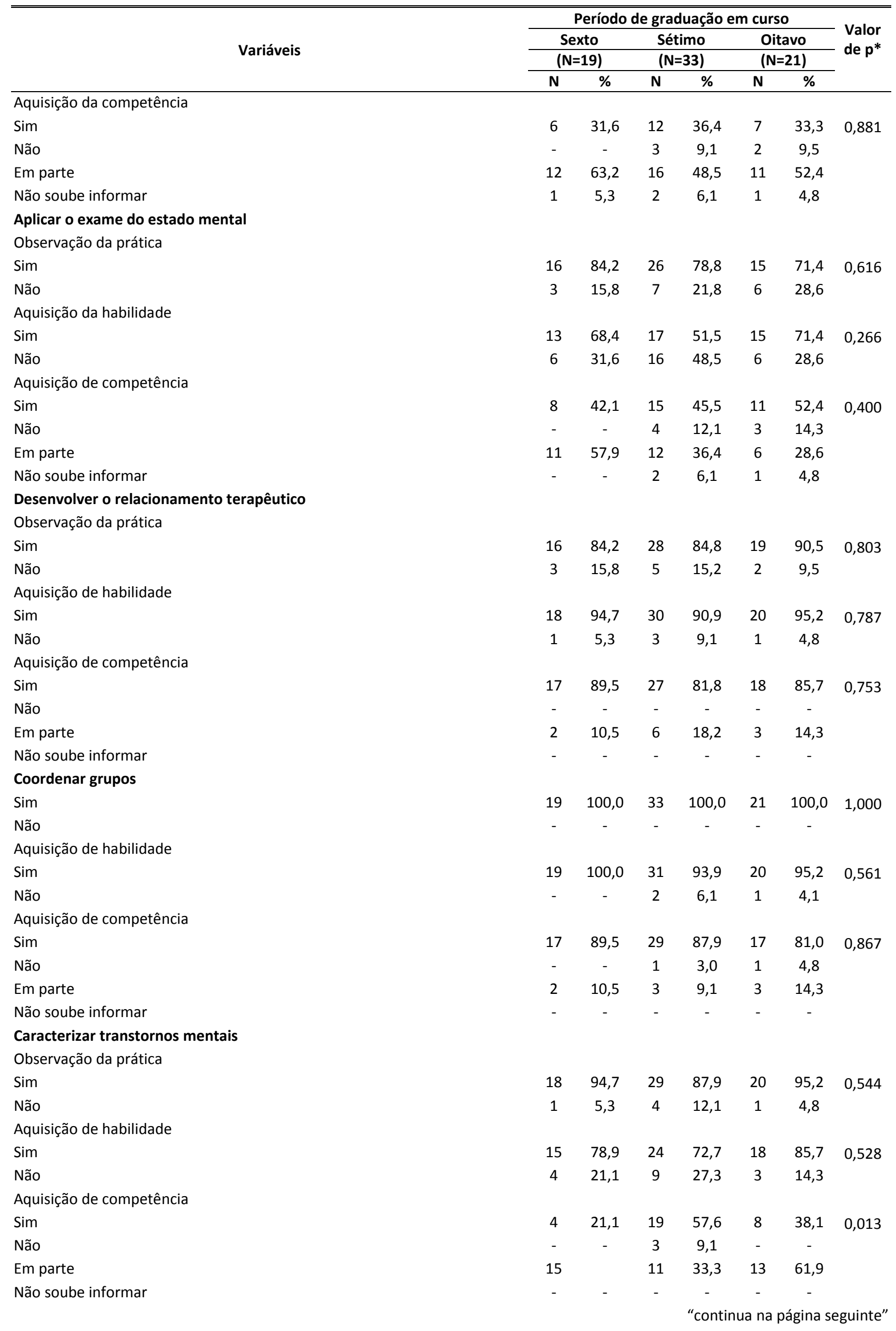




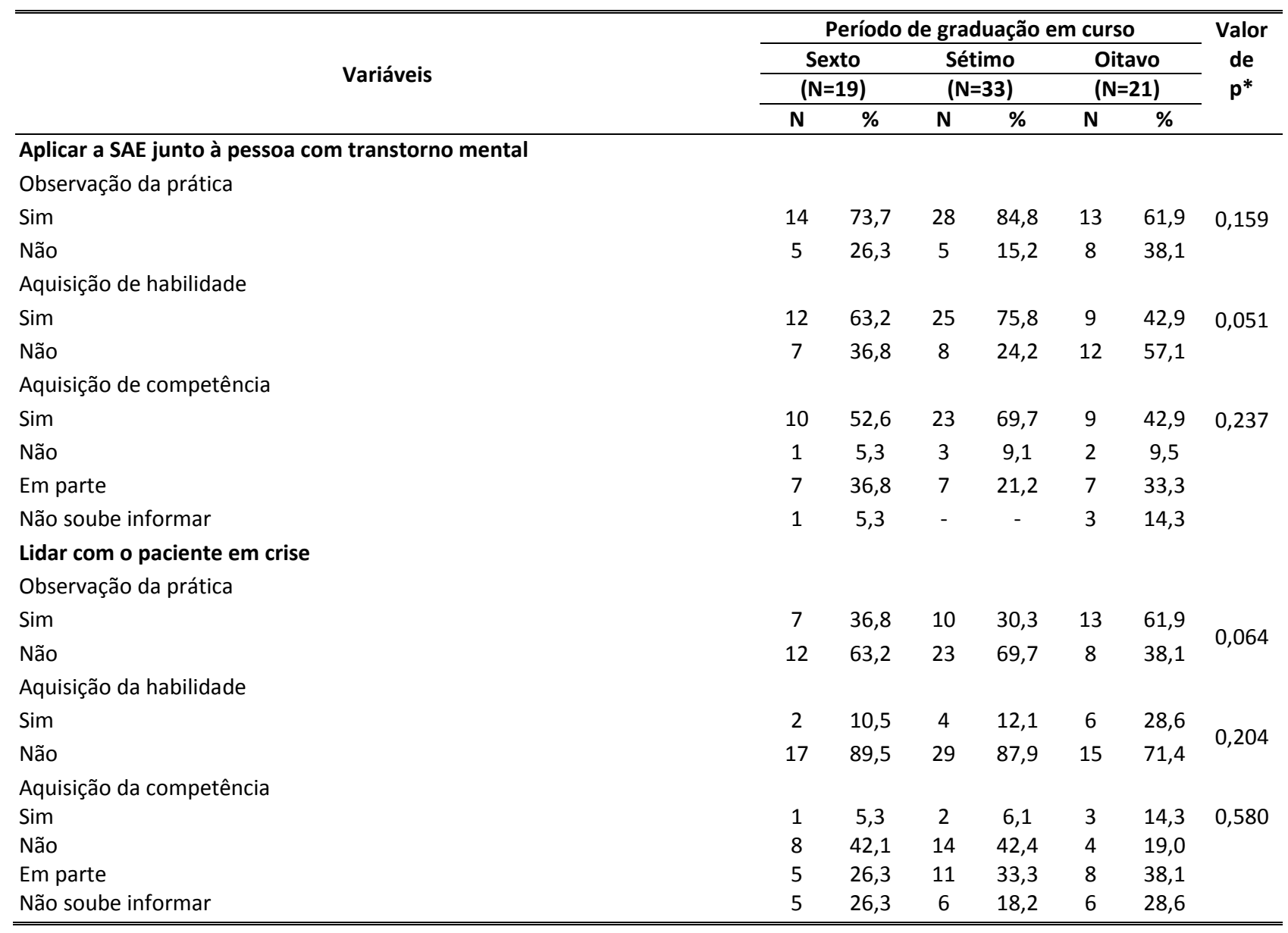

Fonte: Elaborada pelos autores.

*p - Teste Qui-quadrado de Pearson.

Não houve manutenção na aquisição da habilidade de reconhecer preceitos da Política Nacional de Saúde Mental, por parte dos estudantes dos três períodos estudados. Destacou-se a aquisição dessa habilidade no sexto período $(84,2 \%)$, talvez pelo fato de ser na disciplina 'Enfermagem no Processo de Cuidar em Saúde Mental' - ofertada nesse período - que se aborda de forma mais aprofundada o processo de Reforma Psiquiátrica brasileira e a Política Nacional de Saúde Mental vigente. Entre os estudantes do sétimo e oitavo períodos de curso, apenas $45,5 \%$ e $52,4 \%$ respectivamente, avaliaram ter adquirido tal habilidade.

No tocante à variável caracterizar transtornos mentais, foi significante o aumento na aquisição dessa competência do sexto $(21,1 \%)$ para o sétimo período $(57,6 \%)$ de curso, e declínio no percentual de estudante que se sentiam competentes do sétimo $(57,6 \%)$ para o oitavo período $(38,1 \%)$. Tal achado pode apontar a necessidade de uma maior abordagem desse conteúdo, durante o oitavo período de formação.

Quanto à variável, aplicar a SAE junto à pessoa com transtorno mental, apesar do ganho na aquisição dessa habilidade do sexto $(63,2 \%)$ para o sétimo período $(75,8 \%)$, apenas $42,9 \%$ dos estudantes do oitavo período afirmou ter adquirido tal habilidade. Tal achado indica que estudantes do oitavo período de curso sentem-se menos hábeis em aplicar a SAE junto à pessoa com transtorno mental, em comparação com estudantes dos demais períodos estudados, porém não indica que não apliquem a SAE em outros contextos.

Ao se considerar a variável lidar com o paciente em crise de transtorno mental foi significante o aumento de estudantes que observaram a execução de tal prática do sexto $(36,8 \%)$ para o oitavo período de curso $(61,9 \%)$. Acredita-se que esse achado relaciona-se ao fato de, pessoas em crise de transtorno mental, buscarem, mais frequentemente, serviços hospitalares, contexto este no qual estudantes do oitavo período encontravam-se mais presentes. Ainda no que diz respeito a essa variável, identificou-se que, apesar de o estudante do oitavo período ter maior oportunidade de observar a execução dessa prática em comparação aos estudantes de sexto e sétimo períodos, apenas $28,6 \%$ referiram ter adquirido 
tal habilidade e metade destes (14,3\%) afirmou ter adquirido essa competência. Acredita-se que o medo, o receio, o preconceito e o estigma, frequentemente associados ao paciente em crise de transtorno mental, possam interferir no modo como o estudante de enfermagem se relaciona e se comunica com o paciente, contribuindo, significativamente, para tal achado.

De acordo com a Tabela 2, pode-se resumir que houve associação entre períodos de graduação em curso e observação da aplicação da Assistência de Enfermagem em Saúde Mental, no contexto familiar $(p=0,068)$ e aquisição dessa competência $(p=0,059)$; observação da prática, aplicar a Assistência de Enfermagem em Saúde Mental, no contexto comunitário $(p=0,097)$; e aquisição da competência, atuar no nível de atenção primária à saúde mental $(p=0,001)$.

Tabela 2 - Distribuição dos estudantes de acordo com período de graduação em curso e autoavaliação com relação à observação da execução do cuidado de enfermagem em saúde mental por um docente do curso, e aquisição de habilidades e competências para realização do cuidado de enfermagem em saúde mental. Fortaleza, CE, Brasil, 2016. ( $N=73$ ).

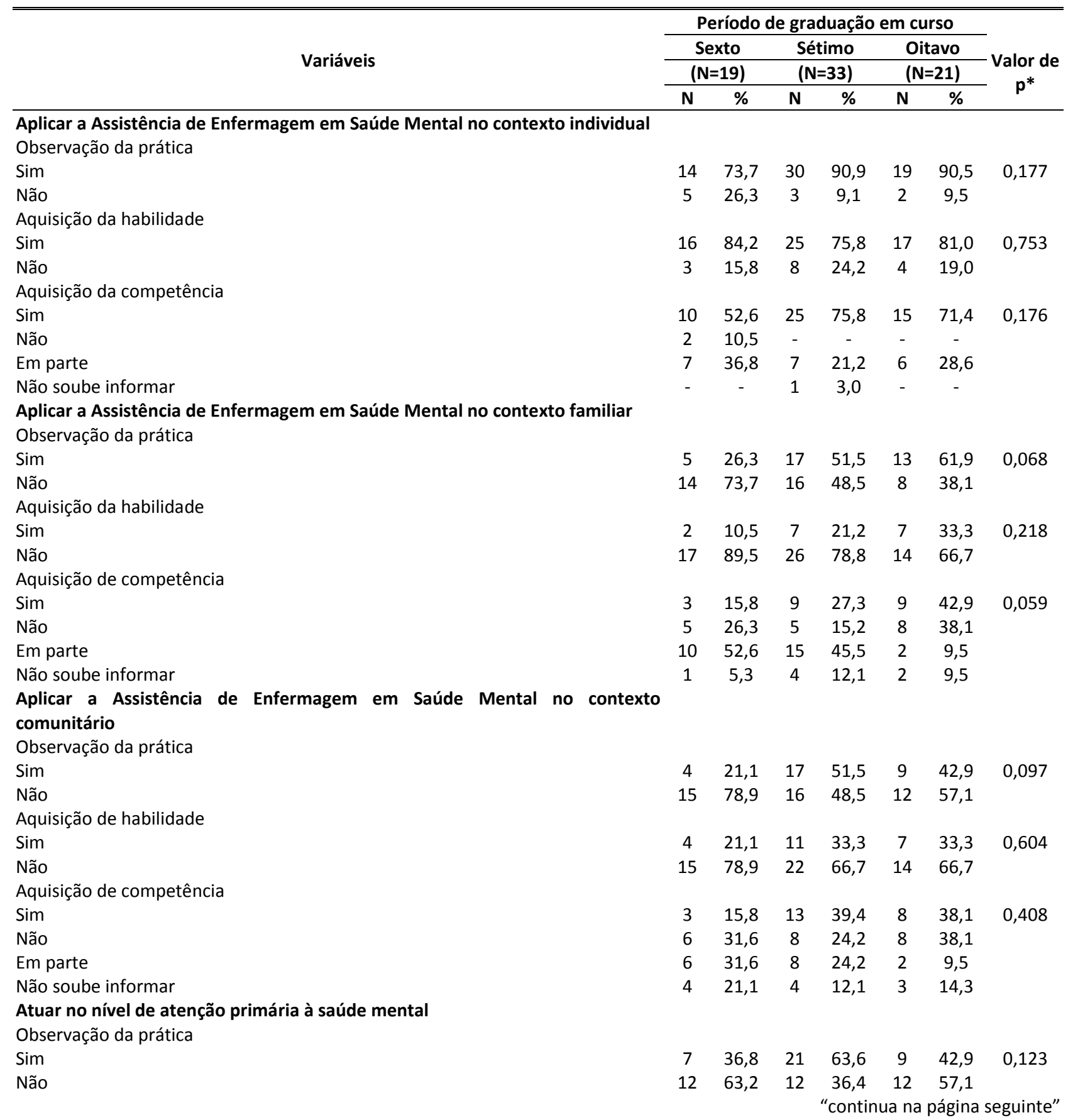




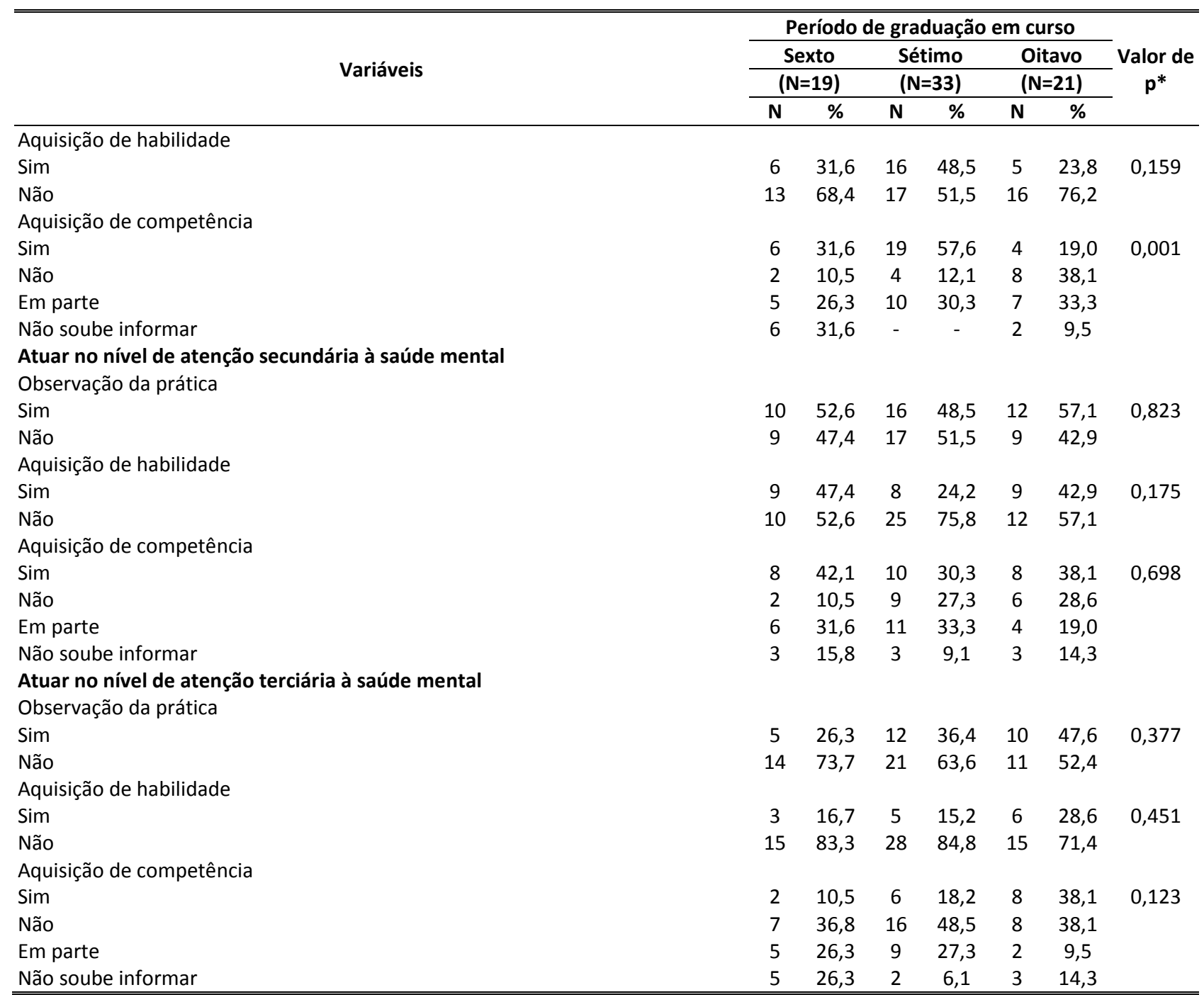

Fonte: Elaborada pelos autores.

* p - Teste Qui-quadrado de Pearson.

Quanto à variável aplicar a Assistência de Enfermagem em Saúde Mental, apesar do aumento gradual no percentual de estudantes que observou a realização de tal prática, no contexto familiar, nos três períodos em estudo (26,3\%; 51,5\%; e 61,9\%; respectivamente), apenas pouco mais da metade de estudantes afirmou ter adquirido tal competência (15,8\%; $27,3 \%$; e $42,9 \%$; respectivamente). Houve aumento do percentual de estudantes que observou a execução da prática aplicar a Assistência de Enfermagem em Saúde Mental, no contexto comunitário do sexto $(21,1 \%)$ para o sétimo período (51,5\%). Porém, houve declínio no percentual de observação dessa prática, por parte de estudantes deste último, para o oitavo período (42,9\%), fato que, talvez, esteja relacionado com uma maior ênfase do conteúdo teórico prático de Enfermagem em Saúde Mental, no contexto hospitalar para estudantes do oitavo período de curso e, consequentemente, menor contato com o contexto comunitário.

A análise da variável, atuar no nível de atenção primária à saúde mental, reforça esse achado, visto que foi significante o aumento percentual de estudantes que avaliou ter adquirido tal competência do sexto $(31,6 \%)$ para o sétimo período do curso $(57,6 \%)$ de enfermagem. No entanto, $38,1 \%$ dos estudantes do oitavo período afirmou que não tinha adquirido tal competência.

Com base na autoavaliação dos estudantes de enfermagem, é possível afirmar que existem significativas lacunas na aquisição de habilidades e competências para a prática de saúde mental, propostas pelo Projeto Político Pedagógico do Curso, ao longo de diferentes períodos do processo formativo.

Até a década de 90 , a ação de enfermagem ocorria, basicamente, em espaços administrativos do hospital psiquiátrico, baseada no modelo 
biológico. Com a Reforma Sanitária, nas últimas décadas do século XX e a consolidação do SUS e Reforma Psiquiátrica, algumas mudanças têm ocorrido no Brasil no que diz respeito às Políticas de Saúde Mental e, com olhar mais atento para assistência extramuros em serviços abertos, docentes têm repensado o conteúdo de suas disciplinas e os cenários de prática para o desenvolvimento de atividades junto aos estudantes $^{(9)}$.

A reorientação da assistência em saúde mental possibilitou não apenas a criação de diversos dispositivos substitutivos aos manicômios, mas, por conseguinte, adequações do conhecimento e das práticas em saúde mental. No entanto, a tentativa de rompimento com o modelo hospitalocêntrico é complexa e exige da enfermagem em saúde mental a quebra do paradigma medicar, conter e vigiar, dando lugar à construção de novas estratégias assistenciais reabilitadoras e promotoras de cuidado efetivo, que solicitam a geração de novos conhecimentos $^{(7)}$.

Para o ensino de saúde mental operar tais transformações é necessário romper com o modelo hegemônico em saúde, desenvolvendo competências para atuar no modelo de promoção em saúde, em sinergia com o modelo psicossocial, sendo o cotidiano da atenção básica um cenário ideal para a constituição de novas competências e habilidades ${ }^{(5)}$.

São imprescindíveis estratégias inovadoras de ensinar e construir possibilidades de cuidados em saúde mental compatíveis com transformações recentes, como: relação interpessoal; projeto terapêutico; inclusão social, reabilitação psicossocial; atenção interprofissional, territorializada e intersetorial. Nessa lógica, a formação de enfermeiros, associada à importância da integração e à reflexão dos saberes e práticas, visa a formar profissionais hábeis e competentes para atuar, conforme o contexto coletivo e social, sustentado no novo modelo de assistência, integral e humanizado $^{(7,10)}$.

A formação por competências e habilidades exige uma pedagogia diferenciada e tem como fundamentos o processo centrado mais na aprendizagem do que no ensino, a valorização do aluno como sujeito da aprendizagem e a construção significativa do seu conhecimento $^{(4)}$.

Acredita-se que, ao se transversalizar preceitos da Reforma Psiquiátrica e da Política
Nacional de Saúde Mental, em disciplinas que abordem o cuidado de enfermagem, nas diferentes etapas do ciclo vital e nos diversos contextos de atuação humana, os estudantes possam ter maiores oportunidades de observação e desenvolvimento de práticas integradas em saúde, favorecendo a aquisição de habilidades e competências.

Entretanto, várias são as fragilidades encontradas no processo formativo em enfermagem em saúde mental, evidenciadas na literatura. Uma delas paira sobre a aplicação da SAE, nesse contexto. São limitadas as experiências que a utilizam e avaliam sua eficácia. Em geral, professores oportunizam a execução de apenas parte dela e as ocasiões que objetivam desenvolvê-la na totalidade de suas etapas restringem-se a experiências localizadas em um contexto institucional particular ou estudos de caso que tomam patologias que se caracterizam na interface de sintomas psíquicos e físicos ${ }^{(1)}$.

Outra barreira para o desenvolvimento de habilidades e competências em saúde mental é a estigmatização da pessoa em sofrimento psíquico. Em sua maioria, estudantes de enfermagem expressam que pessoas com transtorno mental são mais imprevisíveis, violentas e propensas a cometer delitos ou crimes. Tais percepções negativas associam-se, inclusive, com descarte da área da saúde mental na futura carreira profissional. O estigma, o medo e a dúvida podem contribuir de modo que o estudante se distancie do paciente. Nesse ponto, é importante destacar que a formação em enfermagem pode demarcar um espaço que permita apropriação de como se percebe e se reage, para dar conta de redimensionar o olhar diferenciado para a clientela, priorizando práticas mais integradas de ensinar e cuidar ${ }^{(12-14)}$.

O estigma relaciona-se a conhecimentos insuficientes ou estereotipados que levam ao preconceito, à discriminação e ao distanciamento social da pessoa em sofrimento psíquico. Dentre as estratégias fundamentais para mudar tais atitudes estigmatizantes estão aquelas de cunho educativo. O contato com temas pertinentes à saúde mental, durante o processo formativo, pode ser um ponto de partida para abordar as questões da diferença associadas aos problemas de saúde mental, que originam comportamentos discriminatórios e reforçam o estigma. O combate ao estigma na doença mental é um dos principais desafios apontados pela Reforma Psiquiátrica, já que o estigma relacionado às 
doenças mentais associa-se à negação de direitos humanos dos próprios doentes mentais trazendo mais sofrimento ${ }^{(5,15)}$.

Ressalta-se, ainda, que melhorar a competência em saúde mental é importante para a superação do estigma associado aos transtornos mentais. É possível aumentar o conhecimento de estudantes sobre os transtornos, mas isso não implica, necessariamente, a diminuição do estigma. É possível, por exemplo, ter alto grau de competência em saúde mental e não ter, necessariamente, baixas atitudes estigmatizantes com relação ao paciente. Porém, existem evidências de que competência e habilidade, nesse campo, trazem impacto na redução de estigma ${ }^{(16)}$.

Quando o preconceito é vencido, há mais habilidade de interação, visualização do quanto o paciente necessita de atendimento e muita aprendizagem, nesta experiência de cuidado, tanto do paciente quanto do estudante. Muitas vezes, a escuta fica de lado pelo fato de o paciente encontrar-se em crise. Para os estudantes é importante a escuta ativa por ser ela a ferramenta eficiente para ouvir o paciente, tomar providências e personalizar o cuidado ${ }^{(12)}$.

A mudança no pensamento e na postura dos estudantes com relação ao campo da saúde mental, com desconstrução progressiva do medo e do estigma frente ao desconhecido e abertura para compreender o indivíduo em sofrimento psíquico, pode ser possível, a partir da associação entre campo teórico e prático, onde estudantes tentam associar exemplos e conteúdos fornecidos em sala de aula com as experiências vivenciadas na prática ${ }^{(7)}$.

Destaca-se o ensino superior como uma ferramenta crucial para a quebra de paradigmas, propagando a humanização e a garantia do respeito em todas as esferas do cuidado. 0 debate, na academia, acerca do cuidado de enfermagem em saúde mental, pode conduzir o estudante para a conscientização dos princípios propostos pela Reforma Psiquiátrica, propiciando condições para a compreensão do papel do enfermeiro na promoção da saúde mental e coletiva e o despertar do interesse pelo desenvolvimento de habilidades científicas, humanísticas e técnicas que $o$ instrumentalize para a prática profissional ${ }^{(14)}$.

\section{CONCLUSÃO}

Em síntese, no presente estudo, identificaram-se lacunas, no que diz respeito à aquisição de algumas habilidades e competências relevantes para a execução do cuidado de enfermagem em saúde mental, por parte de estudantes em processo formativo. Os resultados evidenciam a necessidade de se adequar o Projeto Político Pedagógico do Curso de Enfermagem, rever o modo pelo qual o ensino está se desenvolvendo e refletir sobre formas de melhorá-lo.

Apesar de trazer resultados importantes no contexto proposto, o estudo apresenta as seguintes limitações: representa a realidade de estudantes de enfermagem em uma única instituição de ensino superior; tem como foco a autoavaliação destes, sem considerar a perspectiva de outros sujeitos importantes no processo formativo; e não confronta os achados com o conhecimento discente, na área de enfermagem, em saúde mental.

Espera-se que tais resultados provoquem na comunidade científica $o$ interesse pelo desenvolvimento de pesquisas, de modo a ampliar a produção científica que aborda a temática e contribuir com o alinhamento dos projetos pedagógicos dos cursos de enfermagem e exercício da prática profissional nesse campo.

\section{REFERÊNCIAS}

1- Vargas D, Maciel MED, Bittencourt MN, Lenate JS, Pereira CF. Teaching psychiatric and mental health nursing in Brazil: Curricular analysis of the undergraduation course. Texto ContextoEnferm. 2018;27(2):11-9. DOI: 10.1590/0104070720180002610016

2- Rodrigues J, Kempfer SS, Lenz JR, Oliveira SN. Influence of curricular reforms in mental health nursing education between 1969 and 2014. Rev Gaúcha Enferm. 2017;38(3):1-9. DOI: 10.1590/1983-1447.2017.03.67850

3- Brasil. Conselho Nacional de Educação. Resolução CNE/CES no 3, de 7 de novembro de 2001. Institui Diretrizes Curriculares Nacionais do Curso de Graduação em Enfermagem. Diário Oficial da União 2001;1:6.

4- Siqueira Júnior AC, Otani MAP. The teaching of psychiatric nursing and mental health in the curriculum by competence. Rev. Min. Enferm. 2011;15(4):539-45. DOI: 10.1590/S0104$\underline{07072013000200016}$

5- Tavares CMM, Gama LN, Souza MMT, Paiva LM, Silveira PG, Mattos MMGR. Specific skills of mental health nurses in undergraduate nursing teaching. Rev Port Enferm Saúde Mental 2016;4:25-32. DOI: 10.19131/rpesm.0137 
6- Lima GZ, Feltrin JÁ, Rodrigues JJ, Buriola AA. Perception of nursing students on mental health home-care: A qualitative approach. J Res Fundam Care 2016;8(2):4255-68. DOI: 10.9789/21755361.2016.v8i2.4255-4268

7- Santos LM, Oliveira RMP, Dutra VFD, Porto IS. The process of knowledge transference: a matter concerning of teaching of psychiatric nursing. Esc Anna Nery Rev Enferm. 2017;21(3):1-

8. DOI: 10.1590/2177-9465-ean-2016-0356

8- Brasil. Conselho Nacional de Saúde. Resolução no 466, de 12 de dezembro de 2012. Dispõe sobre diretrizes e normas regulamentadora de pesquisas envolvendo seres humanos. Diário Oficial da União 2012;1:59.

9- Souza MCBM. The Psychiatric nursing/mental health education: Advances, limitations and challenges. Rev Eletrônica Saúde Mental Alcool Drog. 2016;12(3):139-46. DOI: 10.11606/issn.1806-6976.v12i3p139-146

10- Azevedo AL, Figueiredo NMA, Silva OS, Cardoso MMVN, Porto IS, Araújo STC. Behaviors of nursing students in the learning of communication in mental health. Rev Enferm UFPE 2017;11(10):3878-84. DOI: 10.5205/reuol.12834-30982-1-SM.1110201723

11- Garcia APRF, Freitas MIP, Lamas JLT, Toledo VP. Nursing process in mental health: An integrative literature review. Rev. Bras. Enferm. 2017;70(1):209-18. DOI: 10.1590/0034-71672016-0031

12- Azevedo AL, Araújo STC, Vidal VLL. How nursing students perceive communication with patients in mental health. Acta Paul Enferm. 2015;28(2):125-31. DOI: 10.1590/19820194201500022

13- Vijayalakshmi $P$, Thimmaiah R, Chandra R, Badamath $\mathrm{S}$. Bachelor of nursing student' attitude towards people with mental illness and career choices in psychiatric nursing. An Indian perspective. Invest Educ Enferm. 2015 [citado em 10 mar 2019]; 33(1):138-54. Disponível em: http://www.scielo.org.co/scielo.php?script=sci a rttext\&pid=S0120-

\section{7\&lng=en\&nrm=iso}

14- Barbosa MC, Vasconcelos CR, Oselame GB. The perception of nursing on academic madness. Rev Enferm Atenção Saúde 2016;5(2):3-17. DOI: $\underline{10.18554 / \text { reas.v5i2.1516 }}$

15- Silva LA, Santos I, Tavares CMM. Nursing students' imaginary regarding people with psychological distress: A socio-poetic study. Rev Enferm UERJ 2015;23(4):468-74. DOI: $\underline{10.12957 / \text { reuerj.2015.18917 }}$
16- Ferreira FN, Fernandino DC, Souza GRM, Ibrahim TF, Fukino ASL, Araújo NC, et al. Evaluation of attitudes toward schizophrenic patients among health students. Rev Bras Educ Méd. 2015;39(4):542-9. DOI: 10.1590/198152712015v39n4e01562014

Nota: Artigo extraído da dissertação de mestrado acadêmico intitulada 'Formação do enfermeiro em uma universidade pública no estado do Ceará: competências e habilidades de saúde mental', realizada com o apoio da Coordenação de Aperfeiçoamento de Pessoal de Nível Superior - CAPES.

Recebido em: 25/06/2019

Aprovado em: 14/11/2019

Endereço de correspondência:

Helder de Pádua Lima

Universidade Federal de Mato Grosso do Sul - UFMS. Av Márcio de Lima Nantes S/N, Vila da Barra, Estrada do Pantanal.

CEP: 79400-000 - Coxim/MS- Brasil

E-mail: padua helder@hotmail.com 